\title{
Resultado de un programa de rehabilitación pulmonar sobre la adherencia y la técnica inhalatoria en pacientes con enfermedad respiratoria crónica
}

\author{
Result of a pulmonary rehabilitation program on adherence and \\ inhalation technique in patients with chronic respiratory disease
}

Vilma R. Gómez P. ${ }^{1}$, Carlos E. Aguirre F. ${ }^{2}$, Paola Arévalo M. ${ }^{2}$, Angela Hernández P², Alejandro Casas $\mathrm{H}^{1,2}$.

Forma de citar: Gómez VR, Aguirre CE, Arévalo P, Hernández A, Casas A. Resultado de un programa de rehabilitación pulmonar sobre la adherencia y la técnica inhalatoria en pacientes con enfermedad respiratoria crónica. Rev Univ Ind Santander Salud. 2016; 48(4): 508-515. DOI: http://dx.doi.org/10.18273/revsal.v48n4-2016009 @ (i)

\section{RESUMEN}

Introducción: Los programas de rehabilitación pulmonar deben incluir actividades educativas encaminadas a que el paciente conozca la enfermedad y reciba el adiestramiento que le permita abordar adecuadamente su tratamiento. Objetivo: Analizar la adherencia y la técnica inhalatoria en pacientes remitidos al programa de rehabilitación pulmonar de la Fundación Neumológica Colombiana y evaluar los cambios que se presentaron en éstas al finalizar el programa ambulatorio. Metodología: Estudio no experimental preprueba postprueba en pacientes mayores de 18 años. Se calificó la técnica inhalatoria utilizando la escala EDEN y la adherencia utilizando preguntas adaptadas de la escala de adherencia a la medicación de Morisky. Resultados: Se analizaron 320 pacientes; el porcentaje de pacientes que utilizaba correctamente los dispositivos inhalados al iniciar el programa de rehabilitación osciló entre 9\% y $19 \%$; el error más común en todos los dispositivos fue no expulsar el aire antes de iniciar la inhalación. Después del programa de rehabilitación el porcentaje de pacientes que empleaban una técnica inhalatoria correcta fue en promedio $61 \%$ para inhaladores de dosis medida y $83 \%$ para dispositivos de polvo seco. La adherencia a los inhaladores antes del programa fue $58 \%$, siendo descuido y olvido las principales razones que explican la no adherencia. Al finalizar el programa $86 \%$ de los pacientes eran adherentes. Conclusiones: Un porcentaje bajo de pacientes remitidos al programa de rehabilitación pulmonar aplicaba correctamente la técnica inhalatoria, la instrucción educativa dentro de un programa de rehabilitación pulmonar mejoró la técnica y la adherencia a los inhaladores en un porcentaje importante de pacientes.

Palabras clave: Nebulizadores, cumplimiento de la medicación, resultado del tratamiento, rehabilitación, educación.

1. Universidad del Rosario. Bogotá, Colombia

2. Fundación Neumológica Colombiana. Bogotá, Colombia

Correspondencia: Vilma Rocío Gómez Prada. Dirección: Carrera 13 b 161-85 Bogotá - Colombia Código Postal: 110131. Correo electrónico: vilma.gomez@urosario.edu.co. Teléfono:+1 7428903. 
ABSTRACT

Introduction: Pulmonary rehabilitation programs must include educational activities focused on the acquisition of necessary knowledge and skills to control the disease and approach treatment appropriately. Objective: To analyze treatment adherence and inhalation technique in patients referred to pulmonary rehabilitation program of Fundación Neumológica Colombiana and evaluate changes in them at the end of the outpatient program. Methodology: Pretest - posttest design in patients older than 18 years. The inhalation technique was assessed using the EDEN scale, and adherence was scored with modified questions from the Morisky medication adherence scale. Results: We analyzed 320 patients. The percentage of patients who used inhalers correctly before starting the rehabilitation program ranged between $9 \%$ and $19 \%$; the most common mistake was not exhale the air before starting inhalation. Upon completion of the program the percentage of patients performing a correct inhalation technique ranged between $61 \%$ for metered dose inhalers to $83 \%$ for dry powder inhalers. Adherence to inhalers before the program was $58 \%$, with neglect and forget being the main reasons encountered. It improved in $86 \%$ of evaluated patients. Conclusion: A small percentage of patients referred to pulmonary rehabilitation program applied the inhalation technique correctly; educational instruction in a pulmonary rehabilitation program improved the technique and adherence to inhalers in a significant percentage of patients.

Keywords: Inhalation devices, medication adherence, treatment outcome, rehabilitation. education.

\section{INTRODUCCIÓN}

La administración de medicamentos por vía inhalada permite que éstos se depositen directamente en la vía respiratoria, limitando el potencial riesgo de efectos sistémicos. Para el suministro de medicamentos existen diferentes dispositivos y cada uno requiere de una serie de pasos para lograr su correcta aplicación y garantizar así el depósito pulmonar. Existen además diversos sistemas de evaluación a partir de los cuales se conocen los errores más comunes en la técnica inhalatoria. Los programas de rehabilitación pulmonar buscan alcanzar el mayor grado de independencia de los pacientes y su mayor participación a través del entrenamiento físico, soporte nutricional, soporte psicológico y educación para el automanejo. Este último aspecto debe permitir que el paciente conozca la enfermedad y reciba el adiestramiento que le permita abordar adecuadamente su tratamiento ${ }^{1}$.

Debido a que los errores en la técnica inhalatoria son comunes $^{2}$, uno de los aspectos que se debe incluir en la educación al paciente durante los programas de rehabilitación pulmonar es la instrucción para el uso adecuado de los dispositivos inhalados. Intervenciones de corta duración (una hora) en grupos pequeños (cinco pacientes por grupo) logran ampliar los conocimientos de la vía respiratoria y de los dispositivos inhalados, y mejoran las habilidades para aplicar la técnica inhalatoria adecuadamente sin importar el tipo de dispositivo ${ }^{3}$. Adicionalmente, se ha demostrado que los pacientes con asma que son capaces de usar inhaladores correctamente tienen un mejor control de su enfermedad ${ }^{4}$.
Otro factor importante en el tratamiento de enfermedades crónicas es la adherencia a las intervenciones a largo plazo $^{5}$. Los pacientes con enfermedades respiratorias se ven confrontados a combinaciones de antibióticos, broncodilatadores, antiinflamatorios, oxígeno suplementario, intervenciones como fisioterapia del tórax, ejercicio físico y dejar de fumar, haciendo que el régimen de tratamiento a seguir sea complejo ${ }^{6}$. Específicamente en pacientes con enfermedades respiratorias la falta de adherencia a los inhaladores genera peor control de la enfermedad y mayor número y gravedad de las hospitalizaciones?

Se ha demostrado ampliamente que los programas de rehabilitación pulmonar mejoran la calidad de vida, la tolerancia al ejercicio y disminuyen la disnea ${ }^{8,9}$. Sin embargo, de acuerdo con la revisión realizada en la literatura, no encontramos reportes acerca de resultados de la rehabilitación pulmonar sobre las habilidades para el uso de los dispositivos ni la adherencia a los medicamentos inhalados. Por lo tanto, el objetivo de este estudio fue analizar la adherencia y la técnica inhalatoria en los pacientes remitidos al programa de rehabilitación pulmonar de la Fundación Neumológica Colombiana y evaluar los cambios que se presentaron en éstas al finalizar el programa ambulatorio.

\section{METODOLOGÍA}

\section{Población de estudio}

Se realizó un estudio de antes y después utilizando un diseño no experimental preprueba - postprueba sin grupo control, en pacientes que ingresaron al programa de rehabilitación pulmonar. Durante el programa de 
rehabilitación los pacientes asisten a por lo menos 20 sesiones de entrenamiento físico con una frecuencia de tres sesiones por semana en las cuales se realiza entrenamiento de fuerza y resistencia de los miembros inferiores y de los miembros superiores, educación grupal e individual acerca de la enfermedad y su manejo. De los pacientes que finalizaron el programa de rehabilitación pulmonar se incluyeron aquellos mayores de 18 años con enfermedades respiratorias crónicas con limitación al flujo de aire y que tenían tratamiento al menos con un inhalador al momento de la remisión al programa. Fueron excluidos pacientes sin registro de evaluación de la técnica inhalatoria al iniciar o al finalizar el programa de rehabilitación, asi como pacientes con demencia de cualquier tipo diagnosticada al ingreso al programa.

\section{Evaluación de la adherencia y la técnica inhalatoria}

Para la evaluación de la adherencia a los inhaladores se utilizaron preguntas adaptadas de la escala de adherencia a la medicación de Morisky (MMAS - Morisky medication adherence scale). Anexo $1^{10}$; se consideró que el paciente no era adherente a los inhaladores cuando respondió positivamente a dos o más preguntas. Para la evaluación de la técnica inhalatoria se utilizó la escala propuesta por el grupo $\mathrm{EDEN}^{3}$ con base en una lista de chequeo en la cual se asigna una puntuación numérica a cada paso de la técnica inhalatoria; se definió que la técnica era correcta cuando el paciente realizaba todos los pasos adecuadamente (puntaje total para cada dispositivo en la calificación EDEN), anexo 2.

\section{Actividades y procedimientos}

Todos los pacientes incluidos en el estudio ingresaron al programa de rehabilitación pulmonar entre septiembre de 2009 y junio de 2014. Se revisó la base de datos del programa de rehabilitación pulmonar para identificar aquellos pacientes que cumplieran los criterios de inclusión y exclusión. Posteriormente se revisaron las historias clínicas y se recopilaron los datos demográficos, diagnóstico médico, las respuestas a las preguntas de la escala de adherencia a la medicación y la calificación total de la técnica inhalatoria, tanto al ingreso como al egreso del programa; se obtuvo el porcentaje de pacientes que aplicaban una técnica correcta al inicio y al final del programa de rehabilitación pulmonar y se identificaron los errores más comunes en los pasos para la aplicación de cada dispositivo. Se registraron el número y tipo de intervenciones educativas que recibieron los pacientes. Los datos obtenidos fueron registrados en una base de datos en Excel $^{\circledR}$.

\section{Análisis estadístico}

Se realizó una descripción de la muestra calculando frecuencias y proporciones para las variables de naturaleza cualitativa, promedios y desviaciones estándar para las variables continuas con distribución normal y medianas y rangos intercuartílicos para variables con distribución no paramétrica. Se registró el porcentaje de pacientes que realizaban la técnica inhalatoria correctamente antes y después del programa con cada uno de los dispositivos y se hizo una comparación de muestras relacionadas que permitió encontrar la diferencia entre los dos grupos, con la prueba de Wilcoxon. Todos los valores de $\mathrm{p}$ fueron a una cola y los valores menores de 0.05 se consideraron estadísticamente significativos. Se utilizó el software estadístico SPSS versión 18.

\section{RESULTADOS}

Se analizaron 320 pacientes con edad promedio de 65 años. El $69 \%$ de los pacientes eran mayores de 65 años y el $63 \%$ hombres. Las características demográficas de los pacientes incluidos en el estudio se muestran en la Tabla 1. El 78\% de los pacientes analizados tenían EPOC (enfermedad pulmonar obstructiva crónica), en su mayoría clasificados como EPOC moderada y grave. El $62 \%$ de los pacientes utilizaban un solo dispositivo inhalado, el $32 \%$ dos y el $6 \%$ tres.

Tabla 1. Características de los pacientes que ingresaron al programa de rehabilitación pulmonar

\begin{tabular}{lc}
\hline Características Generales $(\mathbf{N}=\mathbf{3 2 0})$ & \\
\hline Edad, años (Promedio \pm DE) & $69 \pm 10$ \\
Sexo n (\%) & $202(63)$ \\
Masculino & $118(37)$ \\
Femenino & $4,2 \pm 1,5$ \\
Índice de comorbilidad de Charlson, puntaje & \\
(promedio \pm DE) & \\
Variables Espirométricas (Promedio \pm DE) & $2,73 \pm 0,8$ \\
CVF postb2, L & $85 \pm 0,2$ \\
$\%$ CVF postb2 & $1,39 \pm 0,6$ \\
FEV1 postb2, L & $56 \pm 0,2$ \\
\% FEV1 postb2 & $51 \pm 0,14$ \\
VEF1/CVF postb2 & \\
Patología n (\%) & $251(78)$ \\
EPOC & $48(15)$ \\
Asma & $15(5)$ \\
Bronquiectasias & $6(2)$ \\
Bronquiolitis &
\end{tabular}

CVF: Capacidad vital forzada, FEV1: Volumen espiratorio forzado en el primer segundo. postb2: postbroncodilatador. 
Antes del inicio del programa de rehabilitación el inhalador de dosis medida (IDM) sin inhalocámara era utilizado correctamente por el $9 \%$ de los pacientes (22/237), con inhalocámara por el 13\% (7/55); en los dispositivos de polvo seco el porcentaje de pacientes que utilizaban correctamente los dispositivos fue 19\% $(8 / 42)$ con Turbuhaler ${ }^{\circledR}, 15 \%(10 / 65)$ con Accuhaler ${ }^{\circledR}$ y $18 \%(16 / 87)$ con dispositivos monodosis. Tabla 2 . El porcentaje de pacientes que realizaban correctamente cada paso en cada dispositivo inhalado se muestra en la Figura 1. El error más común en todos los dispositivos fue no expulsar el aire seguido de errores relacionados con la maniobra inspiratoria y no hacer la apnea post inspiración. Otros errores comunes fueron no agitar y no esperar para hacer una segunda inhalación en dispositivos IDM.

Tabla 2. Número y porcentaje de pacientes con técnica inhalatoria correcta pre y post rehabilitación pulmonar.

\begin{tabular}{lcc}
\hline TIPO DE DISPOSITIVO & $\begin{array}{c}\text { Pre } \\
\text { Rehabilitación } \\
\text { (n, \%) }\end{array}$ & $\begin{array}{c}\text { Post } \\
\text { Rehabilitación } \\
\text { (n, \%) }\end{array}$ \\
\hline IDM n: 237 & $22(9)$ & $145(61)$ \\
IDM + INH n: 55 & $7(13)$ & $33(60)$ \\
ACCUHALER $^{\circledR}$ n: 65 & $10(15)$ & $52(80)$ \\
TURBUHALER $^{\circledR}$ n: 42 & $8(19)$ & $35(83)$ \\
MONODOSIS $^{\circledR}$ n: 87 & $16(18)$ & $67(77)$ \\
\hline
\end{tabular}

IDM: inhalador de dosis medida, INH: inhalocámara. p $<0,001$

\section{Intervenciones educativas durante el programa}

Todos los pacientes recibieron al menos una instrucción personalizada en el momento del ingreso al programa, $20 \%$ dos, $10 \%$ tres y $9 \%$ cuatro. Dicha instrucción era impartida por la terapeuta del programa y consistía en pedir al paciente que demostrara el uso de los dispositivos inhalados para luego proceder a la corrección de los errores correspondientes; esta intervención se realizaba durante la sesión de entrenamiento con una duración aproximada de 10 minutos. El 21\% de los pacientes recibieron una intervención educativa grupal (en promedio 15 a 20 pacientes por sesión, de aproximadamente 50 minutos a 1 hora de duración) en la que se brindó información acerca de los diferentes tipos de inhaladores y su acción farmacológica y $24 \%$ asistieron a un taller grupal individualizado (máximo cinco pacientes por grupo, de una hora y treinta minutos de duración) enfocado en evaluar y mejorar la técnica inhalatoria, instruir en la identificación de signos de exacerbación respiratoria y en técnicas de higiene bronquial.
Al finalizar el programa de rehabilitación 61\% $(145 / 237)$ de los pacientes que utilizaban el inhalador de dosis medida sin inhalocámara y $60 \%(33 / 55)$ de los pacientes que lo usaban con inhalocámara lograron una técnica correcta $(\mathrm{p}<0.001)$. Entre el $77 \%$ y $83 \%$ de los pacientes que utilizaban dispositivos de polvo seco lograron una técnica inhalatoria correcta al finalizar el programa $(\mathrm{p}<0.001)$. Tabla 2 . Se obtuvo mejoría en todos los pasos de la técnica inhalatoria. Figura1.
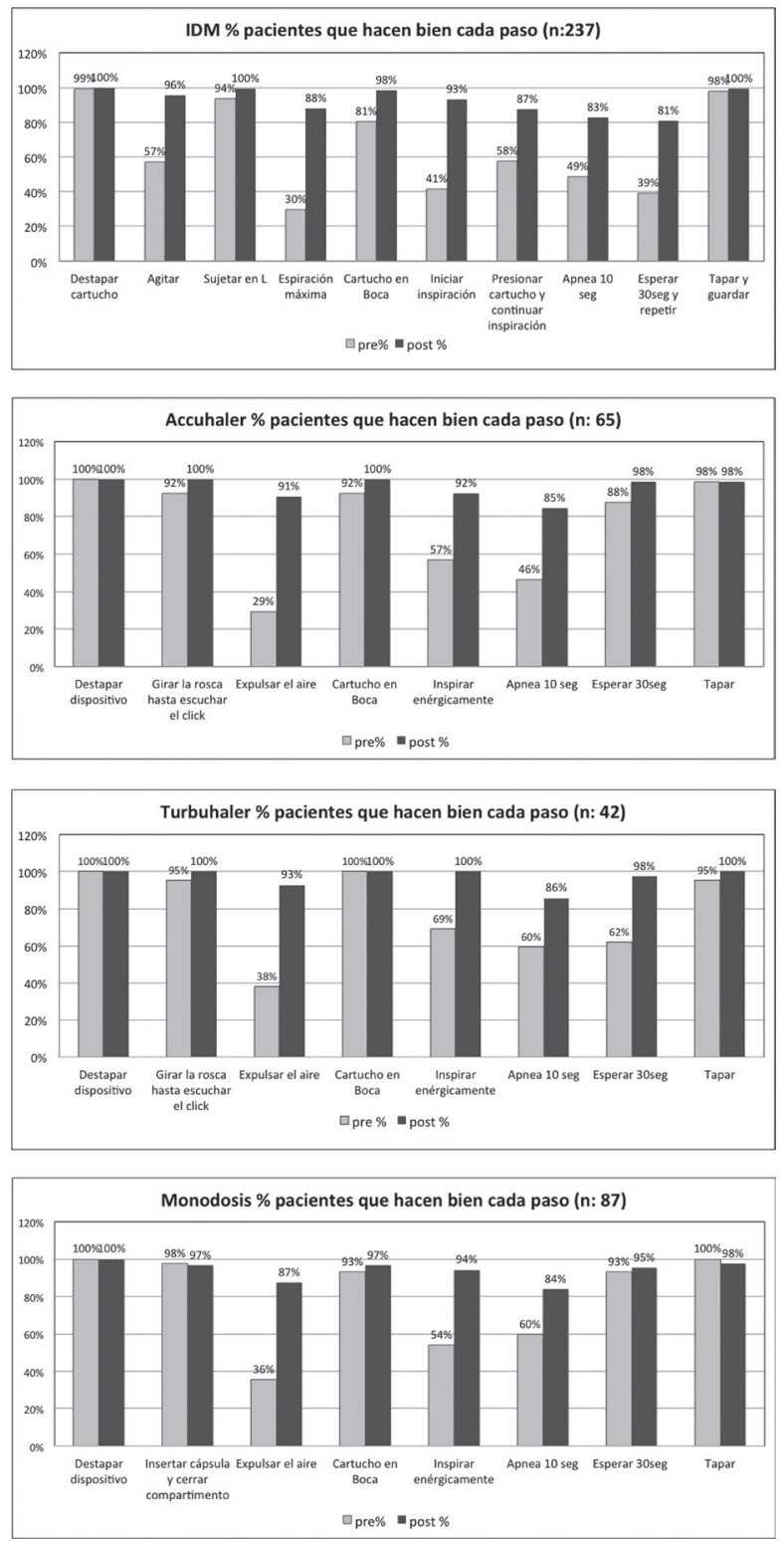

Figura 1. \% de pacientes que realizan correctamente cada paso de la técnica inhalatoria con cada dispositivo pre y post rehabilitación pulmonar.

\section{Adherencia a los medicamentos inhalados}

El 58\% de los pacientes que ingresaron al programa de rehabilitación pulmonar fueron considerados 
adherentes al tratamiento inhalado. Antes del programa, las principales razones asociadas a la no adherencia a los inhaladores fueron descuido y olvido en $39 \%$ y $46 \%$ de los pacientes respectivamente. Al finalizar el programa de rehabilitación pulmonar el $86 \%$ de los pacientes fueron considerados adherentes al tratamiento inhalado. Figura 2.

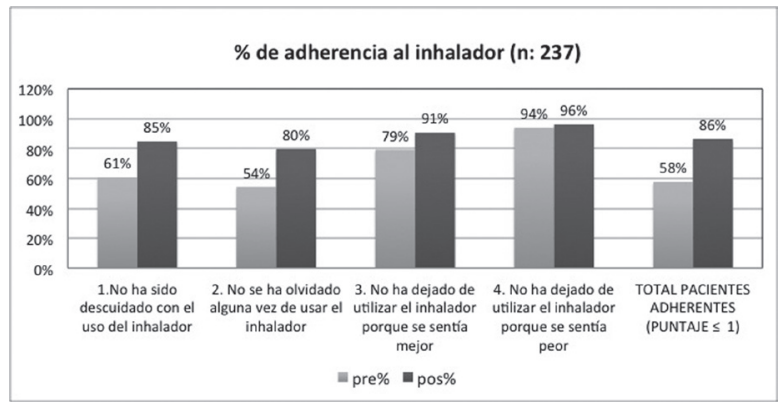

Figura 2. Porcentaje de pacientes adherentes al uso del inhalador.

\section{DISCUSIÓN}

Los resultados de este estudio muestran cómo un programa de rehabilitación pulmonar mejoró la adherencia y la técnica inhalatoria en pacientes que recibieron instrucción acerca del adecuado manejo de los inhaladores. Se encontró que el porcentaje de pacientes que realizaban la técnica inhalatoria correctamente al ingreso al programa fue muy bajo. La información disponible en la literatura en este sentido es variable: en un estudio realizado por BosnicAnticevich, et al. ${ }^{11}$ se encontró que en pacientes que asistían a una farmacía comunitaria solo el 2\% (1/52) tenían una técnica inhalatoria correcta con dispositivos IDM; en otro estudio realizado por Hämmerlein, et al. ${ }^{12}$ el porcentaje de pacientes que tenían una técnica correcta fue $21 \%(160 / 757)$. Además encontramos que el número de pacientes que usaban los dispositivos de dosis medida correctamente fue menor que con otros dispositivos, similar a lo reportado en la literatura ${ }^{2}$.

Al evaluar los cambios con el programa de rehabilitación pulmonar encontramos que en promedio el $60 \%$ de los pacientes que utilizaban dispositivos IDM y el $80 \%$ de los pacientes que utilizaban dispositivos de polvo seco alcanzaron una técnica inhalatoria correcta, lo que fue estadísticamente significativo. Estudios en los que se han evaluado diversas intervenciones han encontrado porcentajes de mejoría variables que pueden alcanzar hasta que el $97 \%$ de los pacientes (31/32 en estudio realizado por Rydman, et al. en 1999) logren una técnica correcta cuando se brinda instrucción verbal y demostración cara a cara² .
Aunque se obtuvo mejoría en todos los pasos de la técnica inhalatoria, no se logró una mejoría de la técnica en el $100 \%$ de los pacientes. Lo anterior puede deberse a diferencias en el tipo y frecuencia de las intervenciones educativas y a que la mayoría de nuestros pacientes son de edad avanzada, lo cual puede afectar la capacidad cognitiva para entender y retener instrucciones dado que algunos estudios han mostrado que a mayor edad aumenta la probabilidad de tener errores en la técnica inhalatoria ${ }^{13}$.

Los errores más comunes en el procedimiento de inhalación encontrados en esta investigación son similares a los reportados en otros estudios ${ }^{3,14-18} \mathrm{y}$ en todos los dispositivos están relacionados con la falta de una espiración máxima previa a la maniobra de inhalación, errores en la maniobra inspiratoria (no realizar la maniobra, no coordinarla con la activación del dispositivo IDM o hacerla muy lenta o muy rápida) y ausencia de apnea post inspiración. Adicionalmente, en los dispositivos IDM otros errores comunes encontrados fueron el no esperar para hacer una segunda inhalación y no agitar el dispositivo. Estos pasos son considerados críticos en la técnica inhalatoria y de hecho son los que reciben una mayor puntuación en la escala $\operatorname{EDEN}^{3,12,16,17}$. Estos errores también se han encontrado en pacientes que acuden a recibir medicamentos en una farmacia ${ }^{11,12}$ $\mathrm{y}$ en pacientes hospitalizados ${ }^{16}$.

Resulta importante establecer, mediante futuros estudios de investigación, el método óptimo para instruir a los pacientes y la frecuencia ideal de instrucción durante un programa de rehabilitación pulmonar, con el fin de garantizar la apropiación de las habilidades que permita dar cumplimento al tratamiento ambulatorio indicado y consiga mejorar la técnica y la adherencia al tratamiento antes de considerar escalonar o cambiar el tratamiento ${ }^{19}$. Así como evaluar las mejores estrategias para mejorar la adherencia a los tratamientos a largo plazo (por ejemplo, medicamentos, actividad física y oxigenoterapia) que por sí misma afecta el control de la enfermedad, problema creciente a medida que aumenta la prevalencia de enfermedades crónicas ${ }^{7}$. También será importante evaluar cuáles son las mejores intervenciones de seguimiento y reevaluación de la técnica inhalatoria una vez el paciente finaliza el programa de rehabilitación ambulatorio puesto que se ha demostrado que las habilidades se deterioran después de un tiempo de recibida la instrucción educativa ${ }^{20}$.

Consideramos que la principal fortaleza del presente estudio es el análisis de un número importante de pacientes en los que se utilizaron herramientas de fácil aplicación y que identifican puntualmente los errores comunes en la 
técnica inhalatoria y los aspectos asociados a la falta de adherencia; estos cuestionarios son útiles para la mayoría de dispositivos disponibles. Sin embargo, a pesar que la rehabilitación de todos los sujetos se realizó en el mismo centro, encontramos diferencias en las características de la instrucción educativa impartida a cada uno de ellos, limitando la posibilidad de conocer cuál o cuáles fueron las estrategias más eficientes para obtener los resultados. Adicionalmente, en programas de atención integral como la rehabilitación pulmonar, resulta difícil controlar todas las intervenciones de todos los miembros del equipo de salud que pudieran influir en los resultados. Cabe anotar que las terapeutas que evaluaron al paciente pudieron ser las mismas que lo intervinieron, lo cual pudo limitar la objetividad en el momento de medir la adherencia y la técnica inhalatoria.

\section{CONCLUSIONES}

El porcentaje de pacientes que realizaban una técnica inhalatoria adecuada al ingreso al programa de rehabilitación pulmonar era bajo; los errores más frecuentes en la técnica inhalatoria coincidencialmente se presentaron en los pasos considerados cruciales en la misma. La educación e instrucción brindada en el contexto de un programa de rehabilitación pulmonar acerca del uso adecuado de los inhaladores puede mejorar la técnica y la adherencia a los medicamentos inhalados en un porcentaje importante de pacientes.

\section{CONSIDERACIONES ÉTICAS}

Este estudio fue aprobado por el Comité de Ética de la Fundación Neumológica Colombiana.

\section{CONFLICTO DE INTERESES}

No existe conflicto de intereses.

\section{REFERENCIAS}

1. Giner J, Donaire F. Educación del paciente respiratorio crónico. En: Güell R, Lucas P. En: Tratado de Rehabilitación Respiratoria. Barcelona: Ars XXI; 2005: p. 149-150.

2. Brocklebank D, Ram F, Wright J, Barry P, Cates C, Davies L, et al. Comparison of the effectiveness of inhaler devices in asthma and chronic obstructive airways disease: a systematic review of the literature. Health Technol Assess. 2001; 5(26): 1-149.

3. Giner J, Macián V, Hernández C. Estudio multicéntrico y prospectivo de "educación y enseñanza" del procedimiento de inhalación en pacientes respiratorios (estudio Eden). Arch Bronconeumol. 2002; 38(7): 300-305. DOI: 10.1016/S0300-2896(02)75222-8.

4. Levy ML, Hardwell A, McKnight E, Holmes J. Asthma patients' inability to use a pressurised metered-dose inhaler (pMDI) correctly correlates with poor asthma control as defined by the Global Initiative for asthma (GINA) strategy: a retrospective analysis. Prim Care Respir J. 2013; 22(4): 406-411. DOI: 10.4104/pcrj.2013.00084.

5. Silva G, Galeano E, Correa J. Adherencia al tratamiento. Implicaciones de la no adherencia. Acta Med Colomb. 2005; 4: 286-273.

6. Kaplan R, Ries A. Adherence in patient with pulmonary disease. En: Hodking J, Celli B, Connors $\mathrm{G}$, editores. Pulmonary rehabilitation guidelines to success. 3a. ed. Baltimore: Lippincott William \& Wilkins; 2000: 347-361.

7. Organización Mundial de la Salud. Adherencia a los tratamientos a largo plazo. Pruebas para la acción. 2004.

8. Ries AL, Carlin BW, Carrieri-Kohlman V, Casaburi R, Celli BR, Emery CF, et al. Pulmonary rehabilitation: Joint ACCP/AACVPR evidencebased guidelines. Chest. 1997; 112(5): 1363-1396. DOI: http://dx.doi.org/10.1378/chest.06-2418.

9. Puhan MA, Lareau SC. Evidence-based outcomes from pulmonary rehabilitation in the chronic obstructive pulmonary disease patient. Clin Chest Med. 2014; 35(2): 295-301. DOI: http://dx.doi. org/10.1016/j.ccm.2014.02.001.

10. Nogués Solán X, Sorli Redó ML, Villar García J. Tools to measure treatment adherence. An Med Interna. 2007; 24(3): 138-141. DOI: 10.4321/ S0212-71992007000300009.

11. Bosnic-Anticevich SZ, Sinha H, So S, Reddel HK. Metered-dose inhaler technique: the effect of two educational interventions delivered in community pharmacy over time. J Asthma. 2010; 47: 251-256. DOI: $10.3109 / 02770900903580843$.

12. Hämmerlein A, Müller U, Schulz M. Pharmacistled intervention study to improve inhalation technique in asthma and COPD patients. J Eval Clin Pract. 2011; 17: 61-70. DOI: 10.1111/j.13652753.2010.01369.x.

13. Melani AS, Bonavia M, Cilenti V, Cinti C, Lodi $\mathrm{M}$, Martucci $\mathrm{P}$, et al. Inhaler mishandling remains common in real life and is associated with reduced disease control. Respir Med. 2011; 105(6): 930-938. DOI: 10.1016/j.rmed.2011.01.005.

14. Melani AS. Inhalatory therapy training: a priority challenge for the physician. Acta Biomed. 2007; 78(3): 233-245. 
Resultado de un programa de rehabilitación pulmonar sobre la adherencia y la técnica inhalatoria en pacientes con enfermedad respiratoria crónica

15. Alamoudi O. Pitfalls of inhalation technique in chronic asthmatics. Effect of education program and correlation with peak expiratory flow. Saudi Med J. 2003; 24(11): 1205-1209.

16. Li H, Chen Y, Zhang Z, Dong X, Zhang G, Zhang H. Handling of Diskus dry powder inhaler in Chinese chronic obstructive pulmonary disease patients. J Aerosol Med Pulm Drug Deliv. 2014; 27(3): 219227. DOI: 10.1089/jamp.2012.1033.

17. Duerden M, Price D. Training Issues in the use of Inhalers. Dis Manag Heal Outcomes. 2001; 9(2): 75-87. DOI: 10.2165/00115677-200109020-00002.
18. Batterink J, Dahri K, Aulakh A, Rempel C. Evaluation of the Use of Inhaled Medications by Hospital Inpatients with Chronic Obstructive Pulmonary Disease. 2012; 65(2): 111-118.

19. Global Initiative for Asthma. Estrategia global para el manejo y la prevención del asma. 2014.

20. Crompton GK, Barnes PJ, Broeders M, Corrigan C, Corbetta L, Dekhuijzen R, et al. The need to improve inhalation technique in Europe: a report from the Aerosol Drug Management Improvement Team. Respir Med. 2006; 100(9): 1479-1494. DOI: 10.1016/j.rmed.2006.01.008

\section{ANEXOS}

Anexo 1. Escala de la adherencia a la medicación de Morisky (adaptada)

\begin{tabular}{lc}
\hline \multicolumn{1}{c}{ PREGUNTAS } & SI \\
\hline 1. ¿Ha sido descuidado a veces en el uso de su inhalador? & NO \\
2. ¿Se ha olvidado alguna vez de utilizar su inhalador? & \\
3. ¿Ha dejado de utilizar su inhalador porque se sentía mejor? & \\
4. ¿Ha dejado de utilizar su inhalador porque se sentía peor? & \\
\hline
\end{tabular}

SI: 1 NO: 0. Paciente adherente: puntaje igual o menor a 1.

ANEXO 2. - Escala adaptada del estudio multicéntrico y prospectivo de "educación y enseñanza" del procedimiento de inhalación en pacientes respiratorios. Estudio EDEN ${ }^{3}$

\begin{tabular}{|c|c|}
\hline TIPO DE INHALADOR & Puntaje \\
\hline \multicolumn{2}{|l|}{ Inhalador de dosis medida* } \\
\hline 1. Destapar el cartucho & 5 \\
\hline 2. Agitarlo & 10 \\
\hline 3. Sujetarlo vertical (forma de L) & 5 \\
\hline 4. Efectuar una espiración máxima & 5 \\
\hline 5. Colocar la boquilla del cartucho en la boca y cerrar completamente & 5 \\
\hline 6. Inspirar lentamente por la boca (lengua en suelo del paladar) & 10 \\
\hline 7. En inspiración, presionar una sola vez el cartucho e inspirar hasta CPT & 10 \\
\hline 8. Apnea - (Mantener la respiración 10 segundos) & 10 \\
\hline 9. Esperar medio minuto entre cada toma y agitar nuevamente el cartucho & 5 \\
\hline 10. Tapar el inhalador y guardarlo en lugar seco & 5 \\
\hline TOTAL & 70 \\
\hline \multicolumn{2}{|l|}{ Inhalador de dosis medida con Inhalo cámara* } \\
\hline 1. Destapar el cartucho & 5 \\
\hline 2. Agitarlo & 10 \\
\hline 3. Acoplar el cartucho en el orificio de la inhalo cámara & 5 \\
\hline 4. Efectuar una espiración máxima & 5 \\
\hline 5. Colocarse la cámara en la boca & 5 \\
\hline 6. Presionar una sola vez el cartucho & 5 \\
\hline 7. Inspirar profundamente el aire de la cámara. - alternativa (respirar de 5 a 10 segundos) & 10 \\
\hline 8. Apnea - (Mantener la respiración 10 segundos) & 10 \\
\hline 9. Esperar medio minuto entre cada toma y agitar nuevamente el cartucho & 5 \\
\hline 10. Tapar el cartucho & 5 \\
\hline TOTAL & 65 \\
\hline
\end{tabular}


Salud Vol.48 No.4 Octubre - Diciembre de 2016

Dispositivo multidosis Symbicort Turbuhaler ${ }^{\circledR *}$

1. Destapar el dispositivo (desenroscar y retirar la capucha del inhalador)

2. Girar la rosca de la parte inferior en sentido contrario a las agujas del reloj, volver a girar la rosca en el sentido de las agujas del reloj. Escuche el clic

3. Expulsar el aire por la boca manteniendo el inhalador apartado de la misma 5

4. Colocar la boquilla entre los dientes y cerrar los labios 5

5. Inspirar enérgica y profundamente 10

6. Apnea - (Mantener la respiración durante 10 segundos) 10

7. Esperar medio minuto entre cada toma y cargar de nuevo 5

8. Tapar el dispositivo

TOTAL 50

Dispositivos unidosis (Handihaler ${ }^{\circledR}$, Breezhaler ${ }^{\circledR}$, aerolizer $\left.{ }^{\circledR}\right) * *$

1. Destapar el dispositivo (Levantar la boquilla del inhalador) 5

2. Insertar la cápsula, cerrar el compartimento y perforar la cápsula 5

3. Expulsar el aire por la boca manteniendo el inhalador apartado de la misma 5

4. Colocar la boquilla entre los dientes y cerrar los labios 5

5. Inspirar enérgica y profundamente 10

6. Apnea - (Mantener la respiración durante 10 segundos) 10

7. Desechar la cápsula completamente vacía 5

8. Tapar el dispositivo

TOTAL 50

Dispositivo multidosis Acchuhaler® (Diskus)*

1. Destapar el dispositivo (Deslizar la tapa del inhalador) 5

2. Bajar la palanca para activar la dosis hasta escuchar un clic 5

3. Expulsar el aire por la boca manteniendo el inhalador apartado de la misma 5

4. Colocar la boquilla entre los dientes y cerrar los labios 5

5. Inspirar enérgica y profundamente 10

6. Apnea - (Mantener la respiración durante 10 segundos)

7. Subir la palanca para cerrar el compartimento de la boquilla y deslizar la tapa 5

8. Tapar el dispositivo

TOTAL 50

*Puntajes adaptados del estudio EDEN.

**Puntaje creado por el programa de rehabilitación pulmonar de la Fundación Neumológica Colombiana. 